\title{
MISSÕES E MISSIONÁRIOS DA PROVÍNCIA DE SANTO ANTÔNIO.
}

FREI VENANCIO WILLEKE, OFM

Diretor do Centro de História Franciscana no Brasil.

A história das missões não comporta detalhes sobre os missionários, exceto um ou outro vulto de destaque. Daí a publicação do seguinte elenco, que por sua vez é incompleto por falta de documentação suficiente. $\mathrm{O}$ número real dos missionários terá sido muito superior ao que figura abaixo, segundo demonstram as lacunas cronologicas das várias aldeias (1) .

As doutrinas da custódia franciscana de Santo Antônio (1585-1619), predecessora da província homônima, ocuparam temporariamente quase todos os religiosos destacadas nas respectivas regiōes, ou seja entre as Alagoas e a Paraiba. Foi sem dúvida nesta época que houve maior zelo na catequese notadamente durante os custodiatos de Frei Melchior de Santa Catarina (1585-1593) e Frei Antônio da Estrela (1602-1606).

E das doutrinas fundadas por Frei Melchior que mais tratam os antigos cronistas franciscanos, embora constem poucos detalhes sobre as atividades e o campo de trabalho de cada missionário. Daí a impossibilidade de enumerar todas as aldeias com os respectivos religiosos.

A entrega das missões franciscanas ao clero secular, por volta de 1619 , obedeceu provavelmente à orientação da corte espanhola. Pois, o mesmo se deu, já no século XVI, com as missões do México. Frei Manuel da Ilha (2) que escreveu em 1621 dá a entender que os missionários depois de terem introduzido os índios na doutrina cristã, confiaram-nos ao ordinário Antônio Teixeira Cabral, para eles

(1). - Frei Venâncio Willeke, OFM, Missões Franciscanas no Brasil, Petrópolis, 1974, p. 33-78 (citado Willeke-Missões).

(2). - Frei Manuel da Ilha, OFM, Narrativa da Custódia de Santo Antônio do Brasil, Petrópolis, 1975, p. 94. O autor escreveu em 1621. 
mesmos encetarem a evangelização de outras tribos pagãs. Como porém o clero diocesano deixasse de corresponder às expectativas de Teixeira Cabral, este pediu aos franciscanos que reassumissem a catequese, mas em vão.

A falta de informes sobre a ocupação das missões reproduzimos a listas das doutrinas:

Missões da Custódia de Santo Antônio 1585-1619.

\begin{tabular}{lcll}
\multicolumn{1}{c}{ Missõess } & fundadas em: & atual Estado: & tribos: \\
Olinda (duas) & 1585 & Pernambuco & Tabajara \\
Itamaracá & 1588 & Pernambuco & Tabajara \\
Itapissuma & 1588 & Pernambuco & Tabajara \\
Ponta de Pedras & 1588 & Pernambuco & Tabajara \\
Almagra & 1589 & Paraíba & Tabajara \\
Praia & 1589 & Paraíba & Tabajara \\
Guiragibe & 1589 & Paraíba & Tabajara \\
Joane & 1589 & Paraíba & Tabajara \\
Mangue & 1589 & Paraíba & Tabajara \\
Siri & 1590 & Pernambuco & Tabajara \\
Tracunhaém & 1590 & Pernambuco & Tabajara \\
Una & 1593 & Pernambuco & Caeté \\
Assunção & 1593 & Paraíba & Tabajara \\
Santo Agostinho & 1593 & Paraíba & Tabajara \\
Piragibe & 1593 & Paraíba & Tabajara \\
Jacoca & 1593 & Paraíba & Tabajara \\
Porto das Pedras & 1597 & Alagoas & Caeté \\
16-18 aldeias & & & \\
anônimas & 1603 & Paraíba & Potiguara \\
\hline
\end{tabular}

Bibliografia: Frei Vicente do Salvador, História do Brasil. São Paulo6, 1975 p. 285 ss; Frei Antônio de Santa Maria Jaboatão, Novo Orbe Seráfico Brasílico. II. Rio de Janeiro. 1858-1862, I, 2 p. 149 ss; Maximiano Lopes Machado, História da Província da Paraíba. Paraíba. 1912 p. 115 ss; Maria do Carmo Tavares de Miranda, Os Franciscanos e a Formação do Brasil. Recife2, 1976 p. 130 ss., além dos autores citados (notas 1 e 2 ).

Em 1624, a custódia de Santo Antônio deu a sua contribuição para a instalação da nova Custódia do Maranhão, cedendo a Frei Cristóvão de Lisboa cinco missionários. Parece que estes se limitaram a introduzir na vida apostólica os confrades recém-vindos do reino por desconhecerem tanto os idiomas indígenas como o método da catequese. Cumprida essa missão, voltaram a Olinda os sacerdo." 
tes Frei Antônio do Calvário, missionário experiente e bom "língua", Frei Manuel Batista, Frei João da Cruz e os irmãos Frei Domingos dos Anjos, Frei Junipero de São Paulo e provavelmente o famoso catequista Frei Francisco do Rosário (3).

Em consequiência da invasão holandesa (1630-1654), já não constam outros trabalhos missionários, senão a assistência religiosa que alguns franciscanos prestaram aos índios acompanhando as tropas nas chamadas entradas. Vários frades tiveram o cuidado de pedir aos mestres de campo certificados do seu apostolado, que em parte se conservaram (4).

\section{Missões da Província.}

Por volta de 1679, a novel Província de Santo Antônio incumbiu-se das primeiras missões entre os silvícolas. O raio de ação estendeu-se pouco a pouco até os sèrtões baianos e pernambucanos, principalmente na bacia do rio de São Francisco. Pois, o governo colonial reconheceu a necessidade de conservar os índios livres no seu habitat primitivo em vez de transferí-los ao litoral, onde estranhavam o clima úmido e a vizinhança inoportuna de muitos brancos e mestiços mal procedidos. Ademais evitava-se a despovoação do hinterland.

A catequese no rio de São Francisco tornava-se particularmente difícil, por causa das frequientes febres. Porisso a província franciscana mantinha sempre alguns religiosos extra-numerários, naquela zona insalubre, para poderem encher as lacunas abertas por graves enfermidades e mortes.

Os cronistas mencionados nas Atas Capitulares (5) devem ter elaborado poucos assuntos a respeito desta fase missionária; pois, Frei Jaboatão the dedica apenas três páginas no seu Novo Orbe S:ráfico Brasílico (6), embora o arquivo provincial oferecesse abundante matéria ainda hoje existente e ele mesmo como cronista da província fosse contemporâneo de muitas doutrinas franciscanas.

(3). - Willeke-Missões, p. 142.

(4). - Arquivo Provincial dos Franciscanos do Recife, (citado APR), $41 \mathrm{fl}, 41$.

(5). - Atas Capitulares da Província Franciscana de Santo Antônio do Brasil, 1649-1893, in "Revista do Instituto Histórico e Geográfico Brasileiro", vol. 286 p. 120 , onde consta a nomeação do cronista Frei Luís da Purificação, em 1718 .

(6) . - Frei Antônio de Santa Maria Jaboatão, OFM, Novo Orbe Seiáfico Brasílico II, Rio de Janeiro, 1858-1862, II p. 801-803 (citạdo Jaboạtão). 
Parece que, depois de encerrada a obra prima de Jaboatão, já não houve quem prosseguisse na elaboração da crônica missionária provincial, embora a catequese sobrevivesse até meados do séculu XIX. E verdade que as missões acompanhavam a decadência da Província de Santo Antônio. Várias missionários secularizaram-se, continuando a exercer a pastoral nas paróquias sertanejas, como padres diocesanos.

De 1760 a 1761 , as missões franciscanas e capuchinhas de Pernambuco foram extintas pelo governo português, sob o pretexto de que os frades não cumpriam as suas obrigações. Mas, os relatórios ainda existentes daquele tempo provam que no meio de religiosos descuidados não faltavam os cumpridores de seus deveres. Jaboatão comenta que o bispo de Olinda, D. Francisco Xavier Aranha concordou com a medida drástica, assim como se adiantara em probir o ensino dos jesuitas (7). Apesar de todas as acusações feitas aos missionários, 121 índios do Pontal-PE chefiados pelo seu cacique Tomás Barbosa, refugiaram-se na missão de Juazeiro da Bahia, pedindo asilo a Frei Francisco de São Sebastião, OFM porque a situação dos aldeados piorara, desde a retirada dos franciscanos do Pontal (8).

Nas missões da Bahia, os franciscanos continuaram até meados do século XIX, quando o número dos índios decresceu cada vez mais e muitas aldeias foram promovidas a paróquias. Em 1863, faleceu o último missionário Frei Antônio da Trindade, contando a aldeia do Saí então 170 anos de catequese franciscana. (Veja na página seguinte).

Como nos escapam os nomes de missionários farnciscanos de várias aldeias, fazemos seguir ao menos o gráfico das missões da Província de Santo Antônio, para oferecer uma visão sobre toda a atividade catequética de quase dois séculos.

Prazos de atividade missionária. Segundo exigia a disciplina rigorosa seiscentista dos franciscanos, cada religioso não passava mais de três anos, em determinada missão, embora mais tarde voltasse à mesma. No tempo da decadência, relaxou tal estatuto, conforme esclarece a lista das aldeias. Poucos missionários se dedicaram à catequese, além de vinte anos, sendo que o governo provincial chamava um ou outro farnciscano para a vida claustral, ao menos por um triênio, ou para confirmá-lo na vocação periclitada ou para confiar-lhe um cargo de categoria.

(7). - Willeke-Missões, p. 84 .

(8). - APR $91 \mathrm{fl} .39$. 
Sobre a vida dos missionários, seu método catequético e atividades em geral, não se acham pormenores, ou porque nunca foram . anotados ou porque se sumiram com as vicissitudes do tempo.

Missões da Província de Santo Antônio 1679-1863.

\begin{tabular}{|c|c|c|c|}
\hline A) Aldeia & B) Orago & $\begin{array}{l}\text { C) Gestão } \\
\text { franciscana }\end{array}$ & D) Tribos \\
\hline 1. Aracapá & S. Francisco & $1702-$ & Cariri \\
\hline 2. Camamu & N.S. do Desterro & $1703-?$ & Cariri \\
\hline 3. Catu & St ${ }^{\circ}$ Antônio & $1719 ?-?$ & Cariri \\
\hline 4. Curral dos Bois & Sto Antônio & $1702-1843$ & Guaimoré \\
\hline 5. Geremoabo & N.S. das Brotas & $1702-1718$ & Tupinambá \\
\hline 6. Itapicuru & N.S. Saude & $1689-1834$ & Tupinambá \\
\hline 7. Jacobina & Bom Jesus & $1706-1859$ & Paiaiá \\
\hline 8. Juazeiro & N.S. das Grotas & $1706-1,843$ & Tamaqueú \\
\hline 9. Massacará & Sma. Trindade & $1689-1854$ & Cariri-Cimbre \\
\hline 10. Massarandupió & St ${ }^{\circ}$ Antônio & $1831-?$ & Tupinambá \\
\hline 11. Pambu & N.S. da Conceição & $1702-?$ & Tamaqueú \\
\hline 12. Piagui & Sta. Cruz & $1706-?$ & Cariri \\
\hline 13. Rodelas & S. João Batista & $1697-?$ & Porcá-Rodela \\
\hline 14. Saí & N.S. das Neves & $1697-1863$ & Cariri-Cimbre \\
\hline 15. Salitre & S. Gonçalo & $1703-?$ & Cariri \\
\hline 16. Aricobé & N.S. da Conceição & $1739-1860$ & Cariri \\
\hline 17. Coripós & N.S. do Pilar & $1702-1761$ & Tapuia \\
\hline 18. Pajeú & St' ${ }^{\circ}$ Antônio & $1741-1761$ & Cariri \\
\hline 19. Pontal & N.S. dos Remédios & $1705-1761$ & Cariri \\
\hline 20. Sorobabé & N.S. do 0 & $1702-1761$ & Tapuia \\
\hline 21. Una (Iguna) & S. Miguel & $1679-1742$ & Caeté \\
\hline 22. Unhunhum & N.S. da Piedade & $1705-1761$ & Tapuia \\
\hline 23. Palmar & $\mathrm{St}^{\circ}$ Amaro & $1695-1699$ & Caeté \\
\hline 24. $\mathrm{St}^{9}$ Amaro & N.S. da Vitória & $1679-1761$ & Caeté \\
\hline 25. Cariris & N.S. do Pilar & $1705-1724$ & Cariri \\
\hline
\end{tabular}

NB. 1-15 Bahia; 16-22 Pernambuco; 23-24 Alagoas; 25 Paraíba. As missões 1-3, 10-13 e 15 passaram poucos anos com os franciscanos. - Catu não aparece nos documentos franciscanos, e sim nos Documentos Históricos, vol. 55, p. 268. - Curral dos Bois não se deve confundir com a missão homônima dos Jesuitas, entre Sentosé e Malhadinhas.

O ensino elementar que os franciscanos ministravam aos seus aldeados consta, em particular na primeira fase missionária de 1585 a 1619 , enquanto depois de 1679 não é mencionado nos documentos coevos, embora ao certo prosseguisse. Igualmente o aprendizado de artes e ofícios é silenciado, podendo porém concluir-se de algum mo- 
do dos inventários de ferramentas das aldeias e da praxe do ensino profissional adotado em todas as missões do Brasil.

A seguir, apresentamos as missões com os religiosos encarregados delas. As listas baseiam-se sobre as tábuas capitulares e os livros de óbitos da província. Alguns nomes foram encontrados em livros paroquiais do sertão baiano e em outras fontes. Muitos missionários continuam anônimos conforme provam as lacunas. $\mathrm{O}$ fato de um missionário aparecer nas tábuas capitulares ainda não prova que realmente exerceu o munus na respectiva aldeia. Tal prova se obtem pelas repetidas assinaturas lançadas nos livros de batizados e casamentos.

\section{AS ALDEIAS E SEUS RESPECTIVOS MISSIONÁRIOS.}

1). - Arăcapá-BA. 1702-? (9).

Apolinário da Transfiguração, 1724 .

2). - Aricobé-PE (BA). 1739-1860.

Tomás da Conceição, 1738 (10)

Apolinário đa Transfiguração, $\pitchfork 1742$

Rafael da Conceição, 1742 (11)

Miguel de Sta. Rosa, 1744, 47, 49, 51, †51 (12)

Gabriel da Vitória (batisa 1746) (13)

Claudio de Sto Antônio, 1748/54

Silvestre de Jesus e Maria, $\$ 1763$

José Maria de St९ Inácio, 1766/9

José do Egito, 1769/72, 74/7

Manuel dos Querubins, 1772

Aleixo de Sta. Isabel, $1777 / 80$

José de Jesus Maria Lima, $1778 / 80$

a

(9). - A interrogação (?) indica a incerteza quanto à duração de várias missões. Adotamos as siglas convencionais dos Estados.

(10). - Arquivo Histórico Colonial 14, 2, onde constam todas as nomeações de missionários feitas em 1738, razão por que não as repetimos. Por via de regra as nomeações eram feitas ao ensejo dos capítulos provinciais e publicadas nas chamadas tábuas capitulares. A cada ano indicado corresponde, pois, a tábua capitular da mesma época, dispensando as citações desses documentos existentes no APR.

(11). - Livro de Batizados da Paróquia de Säo Francisco da Barra, 1744 (citado Batizados-Barra).

(12). - A cruzinha $(\dagger)$ significa que o missionário faleceu, na respectiva aldeia e no ano citado.

(13). - Batizados-Barra 1746, fl. 41v, 
Henrique de S. Joaquim, 1780

Henrique de St 9 Inácio, 1780/3

Joaquim de S. José e Santana, 1780/3, 89/98

José de S. Bernardino, 1787

João do Espírito Santo Graça, 1798

Antônio da Porciúncula II, 1816/9

Luís dos Querubins, 1819

Florentino da Sagrada Família, 1820/29, 1837/40

Antônio dos Anjos, 1829/34

Antônio do Desterro Padilha, 1834/5

Manuel da Natividade Nogueira, 1835

Luís do Monte Alverne, 1840

Antônio de Sta. Clara, 1844

Manuel de Sta. Ludovina, 1849/50

José de Sta. Engrácia, 1850

José de S. Cândido, 1852

Lourenço da Imaculada Conceição, 1854/60

Francisco da SS. Trindade, 1860.

3) . - Coripós-PE. 1702-1761.

Pantaleão da Porciúncula, 1712

José de Jesus Maria Colares, 1735 (14)

Valério de Sta. Rosa, 1738

Antônio das Chagas Azevedo, †1741

Cláudio de St ${ }^{\circ}$ Antônio, 1743/8

Manuel de S. Boaventura, 1755/60

Hermenegildo de S. José, 1760 (15).

4). - Curral dos Bois-BA. 1702-1843 (16)

Pantaleão Batista, 1710

Antônio das 11.000 Virgens, 1725 (17)

Hugolino de St ${ }^{\circ}$ Antônio, antes de 1730

João Batista, (C) antes de 1730

(14) - - A tábua capitular de 1735 falta no APR, existe porém como ms. no Instituto Histórico e Geográfico Brasileiro, arquivo 2, 4, 8 fl. 269r270r. Esta citação vale pois para todas as nomeações de 1735 .

(15). - Coleção do Barão de Studart 5.1. $17 \mathrm{fls} .142 \mathrm{r}$ (ms). Frei Hermenegildo de São José acabava de tomar posse da missão de Coripós, quando uma comissão do governo colonial fiscalizava a aldeia e tomava o inventário de todos os bens. - O Barão de Studart não indica a fonte (citado Studart).

(16) - Curral dos Bois mudou de nome várias vezes, chamando-se por último Glória; a cidade foi transferida para outro local, por causa da recente represa do Moxotó.

(17). - Arquivo Histórico Colonial - Bahia - Documentos avulsos cx. 32,17 . VII. 1725 . 
Arcângelo da Encarnação (M) 1735 (18)

Francisco de Assis (C) 1735 (19)

Eusébio dos Prazeres (M) 1738

Rafael da Purificação (C) 1738

Antônio de Santana Dinis, 1741, 55

Antônio da Piedade, 1743/8

João dos Mártires, 1749/52

Mateus de Sta. Catarina, 1751/77

Faustino do Paraíso, 1760/1

Hermenegildo de S. José, 1761/6†

Cassiano de Santana, 1768/72

Agostinho da Assunção, 1769/74

Antônio de S. José Piedade, 1774/81† (20)

Elias de Sta. Teresa, 1780/3

Francisco de Sta. Clara, 1782/3

Henrique de St? Inácio, 1785

Joaquim da Purificação Borges, 1787/9

Melchior de St' Antônio, 1790/8

Antônio da Apresentação, 1798-1801, 1802/10, 1812 ?

João do Espírito Santo Graça, 1801/2

Antônio do Desterro Padilha, 1810/20, 31

Domingos da Sta. Cruz Moreira, 1820/4

Antônio de Sta. Clara, 1824/32, 34/7, 41/4.

5). - Geremoabo-BA. 1702/18.

Pantaleão Batista, 1710 (21).

6). - Itapicuru-Ba. 1689-1834.

Lázaro da Purificação, 1697

Rufino do Deserto, 1697

Antônio de Jesus, 1710

Nazário do Salvador, 1712

Manuel de St' Antônio Bexiga, 1715, 21

Antônio da Piedade, 1727, 29, 30, 32, 33, 35, 36, (C) $38,41 / 2$

José de Jesus Mária Colares, 1729

(18). - $\mathrm{M}$ - significa missionário superior.

(19). - C - quer dizer missionário companheiro ou súdito.

(20). - Documentos Históricos da Biblioteca Nacional, vol. VII, p. 248 (citado Documentos Históricos) .

(21). - Como missionário de Curral dos Bois, Frei Pantaleão Batista regia ao mesmo tempo a missão de Geremoabo. Cf. APR 1 p. 219. 
Arsênio da Madre de Deus (M) 1735

Manuel da Trindade (M) 1738

Antônio do Paraíso, †1742

Francisco de S. Francisco $1752 / 60$

Manuel de Sta. Teresa, 1763/66 0. III, 69, 79/80, 82, 83

Agostinho da Assunção, 1766/9

Alberto da Conceição, †1766

Boaventura de Jesus Maria José, 1773

Cassiano de Santana, 1774, 76/8 (22)

Manuel de S. Boaventura, 1776/83t

José de Sta. Maria Lima, $1777 ?$

Patrício de Sta. Helena, 1780/91

Plácido de Sta. Helena, 1782

Caetano de S. Joaquim, $1793 / 6$ (23)

Reginaldo da Conceição, 1796/9 (24)

Manuel de Jesus Maria Batista, 1801/2 (25)

Francisco de S. José Sousa, 1802/14

Antônio de Sta. Clara, 1814/20

Dionísio da Conceição, $1820 / 4$

Antônio dos Anjos, 1824/7

Manuel de Sto Antônio, 1828

Sebastião de St' Antônio Marques, 1828/30

Francisco de Sales, 1831/4.

7). - Jacobina-BA . 1706-1859.

Lourenço de Jesus Maria, 1705 (26)

Manuel da Madre de Deus, 1722

João da Natividade Misericórdia, 1722

Francisco das Neves Caguende, 1731, (M) 35, (C) 38

Manuel das Chagas II, (C) 1733, (M) 38

Miguel da Transfiguração, (C) 1735

Custódio de Jesus, 1741 (o único irmão)

Atanásio de Santana, 1752

Florêncio da Conceição, (C) $1760 / 73$

Manuel dos Prazeres, (C) 1766,69

(22). - Documentos Históricos, VII, p. 248.

(23). - Livro de Batizados da Paróquia de Itapicuru de Cima, registra batizados feitos por Frei Caetano.

(24). - Ibidem, Frei Reginaldo de junho de 1798 a março de 1799.

(25). - Ibidem, Frei Manuel de abril de 1800 a outubro de 1801 .

(26) . - Documentos Históricos, vol. 41, pp. 20, 123, 168, 185. Enquanto a província franciscana apenas em 1706 assumiu oficialmente a missão do Bom Jesus da Glória de Jacobina, Frei Lourenço já trabalhava lá desde 1705 . Tais casos se repetem em outras missões. 
Teodósio da Conceição, (M) $1774,76,77,80,82, \nmid 87$

Bento da Natividade, (C) $1776 / 80$

Francisco de St' Antônio, 1780/2

Teodorico da Cruz, 1787

Inácio de Sta. Maria de Jesus Dias, 1789/1801† (27)

Francisco de S. Pedro e Paulo, 1801/5, 08/10, 11/4

Jacinto de Sta. Eufrásia, 1801

Domingos de S. José Lobo, 1807

José da Encarnação Silvestre, 1810/1, 13/20, 27/8, 29/31, $34 / 40,41 / 59 \dagger$

Manuel de S. Boaventura, $1820 / 26$

Dionísio da Conceição, 1828/29

Manuel de Maria Santíssima, 1831/2.

8). - Juazeiro-BA . 1706-1843.

Manuel da Madre de Deus Sampaio, antes de 1726 (28)

Manuel da Madre de Deus Chaves, (M) 1735, 1738

Valério de Sta. Rosa, (C) 1735

Miguel de Sta. Rosa, (C) 1738

Maurício de S. Francisco Macedo, antes de 1739

Manuel das Chagas II, 1739

Antônio de Santana Dinis, 1743

Ivo do Sacramento, 1748

Francisco de S. Sebastião, 1755/57, 68,77/79 (29)

Libório da Assunção, $† 1760$

José de S. Gabriel, 1762/65

Manuel de S. Boaventura, 1764/74

Manuel dos Querubins, 1767, 1774/77

Luciano das Neves, $1780,82-83,85$

Francisco de Sta. Clara, 1780/2, 83/7, 89/90

Joaquim de S. José e Santana, 1787, 1802, 04/7†

Inácio de Sta. Maria Jesus Dias, 1787

Francisco de S. Domingos, $1793 / 5 \dagger$

Domingos do Rosário Aragão, 1796/98†

Melchior de St' Antônio, 1799

João de Santana, $\nmid 1800$

Joaquim de Santana, 1804/8

João de S. José Cupertino, 1808/12†

(27). - O Livro de Batizados da Paróquia de Jacobina registra batizados ministrados por Frei Inácio em 1796 e 1797.

(28). - Documentos Históricos, vol. 72, p. 289. - Frei Manuel fez o frontispício da igreja missionária do Juazeiro de pedra e cal.

(29). - Documentos Históricos, vol. VII, p. 248. 
Antônio do Rosário, 1813/6†

José de S. Vicente, 1816/20, 35

José de Sta. Gertrudes, 1820/3 (30)

José de Sta. Ursula, 1820 (31)

Antônio de Porciúncula II, 1821/2 $\dagger$

José da Encarnação Silvestre, 1823/6 (32)

Manuel da Ressurreição, 1827/28, 29/30, 31/32

Sebastião de St $^{\circ}$ Antônio Marques, 1834/5, 37/40 (33).

9). - Massacará-BA. 1689-1854.

Daniel de St ${ }^{\circ}$ Agostinho, 1706

Manuel da Madre de Deus Sampaio, $\dagger 1727$

Rufino do Deserto, $\dagger 1732$

Antônio de Nazaré, 1733

Brás de Santana, (M) 1735

João de Sta. Maria, (C) 1735

Lopo de Sta. Rita, antes de 1739

Antônio do Paraíso, (M) 1738

Manuel de Santana Fontes, (C) 1738

Januário de $\mathbf{S t}^{\circ}$ Antônio, $\uparrow 1748$

José de Sta. Joana Caixeira, $\$ 1752$

Antônio de Santana Dinís, 1758/72

Francisco de S. Francisco, 1763/70

Pedro da Assunção, $† 1763$

Manuel de Sta. Teresa, 1771/9

. Manuel de S. Boaventura, 1774/6, 77 (34)

Francisco de Sta. Clara, 1776/77

Carlos de S. José, 1780/2

Bento da Apresentação, 1782/5

Bento da Natividade, 1782/4

Francisco dos Prazeres, 1785/90

João de S. Caetano Nunes, 1790/1†

Francisco da Conceição Solano, 1792/3

Francisco de S. José Sousa, 1793/1802

Manuel da Purificação, 1802/14

Manuel de S. Vicente, 1814/7

José de Jesus Maria Lopes, 1817/9

(30). - Livro de Batizados da Paróquia do Juazeiro, registra batizados feitos por Frei José de Santa Gertrudes, de 1821 a 1823 . Como religioso português o frade declarou-se contrário à Independência do Brasil.

(31). - Ibidem, batizados feitos por Frei José de Santa Ursula em 1820. a 1826.

(32). - Ibidem, batizados feitos por Frei José da Encarnação de 1823

(33). - Ibidem, batizados feitos por Frei Sebastião de 1832 a 1840.

(34). - Documentos Históricos, vol. VII, p. 248. 
José da Anunciação, 1819/20, 29/31

Antônio do Desterro, 1820, 24, 41

José de Sta. Ursula, 1825/8

Manuel da Ressurreição, 1828/9

Florentino da Sagrada Família, 1831/2, 34/5, 40/1

Francisco de Sales, 1835

Antônio dos Anjos, 1837/8

Francisco da Solidade, 1838/40

José da Encaranção Silvestre, 1840/1

João de Sta. Delfina, 1846/7

Sebastião de St ${ }^{\circ}$ Antônio Marques, 1847/52

Roberto de S. Gonçalo, 1849.

10). - Massarandupió-BA. 1831-?

José da Piedade, 1831.

11). - Pajeú-PE. 1741-1761.

João de Sta. Rosa, 1741

Lino da Cruz, 1741 (35)

Antônio de Sta. Escolástica Picão, 1755/60 (36).

12). - Palmar-AL. 1695-1699.

Damião das Chagas, 1697

Manuel da Encarnação, 1699 (37).

13). - Рambu-BA. 1702-?

José dos Santos Gama, $\$ 1750$

Antônio Sta. Clara, 1821, 23, 25, 27

Antônio do Desterro Padilha, 1831/34

Manuel da Ressurreição, 1828/9.

14). - Pontal-PE. 1705-1761.

Antônio de Sta. Rosa, 1715 (38)

Francisco da Conceição, (M) 1735

Francisco de Sta. Ângela, (C) 1735

Matias da Encarnação, (M) 1738

Inácio da Conceição, (C) 1738

(35). - Willeke - Missões, p. 84.

(36). - Studart, f1. 45r. referindo-se ao ano de 1760, quando da fiscalização das missões.

(37). - Arquivo Histórico Ultramarino - Pernambuco: avulsos cx. 10, 1698-1700.

(38)..-Documentos Históricos, vol. 42, p. 275, onde esclarece que Frei Antônio de Santa Rosa foi acusado sem razão. 
Emiliano de Jesus, $\uparrow 1749$

José de S. Gabriel, (M) 1758/60.

15). - Rodelas-BA . 1697-?

Antônio de Sta. Clara, 1821 (regente).

16). - Saí-BA. 1697-1863.

Francisco dos Anjos, 1697

Francisco da Piedade, 1697

Luis da Penha, 1705 (39)

Melchior da Madre de Deus, 1715

Manuel da Madre de Deus Sampaio, 1722 (40)

Francisco da Conceição Vage, (M) 1735

Manuel da Chagas, (C) 1735

Antônio da Trindade I, 1738

Manuel da Madre de Deus Cotinguiba, 1738

Vivaldo de S. José, antes de $1739,+1755$

Antônio da Purificação Fialho, 1752/8

Florêncio da Conceição, 1758

Bento de S. José, 1760/3†

Sebastião de Santana, $\dagger 1767$

Manuel dos Prazeres, 1768

José Maria de St ${ }^{9}$ Inácio, 1769/71†

José do Egito, 1772/4

Cassiano de Santana, 1772

Antônio de Sta. Úrsula, 1777/80

Francisco dos Prazeres, 1777, 1780

Bento da Natividade, 1780, 1782

Domingos da Assunção, 1785/96

Antônio da Apresentação, 1796/8, 1811/3

Joaquim de S. José e Santana, 1798/1804

Domingos de S. José Lobo, 1802/7

Francisco de S. Pedro e Paulo, 1807/8, 10/1, 13/4

Antônio do Deterro, 1808, 25, 29

João do Bom Sucesso, 1814/7

Francisco do Bonfim, 1817/20

José de Sta. Ursula, 1820, 25

Domingos da Sta. Cruz, 1827/9

Sebastião de St ${ }^{\circ}$ Antônio Marques, 1831, 35, 41

(39). - Ibidem, vol. 40, p. 341.

(40). - Afonso Costa História da Jacobina in "Jornal do Comércio", Rio de Janeiro, 31. VIII. 1952. 
Manuel da Natividade Nogueira, 1834/5

Antônio de Sta. Clara, 1837/41

José de S. Vicente, $1847 / 8$

Antônio da Puríssima Conceição, 1849, 50

José das Dores Barata, 1851/2

José do Coração de Maria, 1852

Francisco de Sta. Guilhermina, 1854/6

Cândido da Virgem Maria, 1856/9

Francisco da Purificação, 1859/62

Antônio da Trindade II, 1862.

17) . - Salitre-BA. 1703-?

Daniel de St? Agostinho, 1705 (41).

18). - St. Amaro-AL. 1679-1761.

Francisco dos Anjos, 1679 (42)

Brás de Sta. Maria, (M) 1681

José da Sta. Cruz, (C) 1681

Luis da Conceição, (C) 1697

Damião das Chagas, (M) 1701

Raimundo de Sta. Margarida, (M) 1721, 38

Miguel de Sta. Catarina, (C) 1726/7, 1735/8

Amaro da Conceição, 1729

Antônio do Paraiso, (M) 1735

Rodrigo dạ Ascensão, (M) 1738.

19). - Sorobabé-PE. 1702-61.

Lucas da Assunção, (M) 1735/40†

João Batista da Conceição, (C) 1735

Arcângelo da Encarnação, (C) 1738

Miguel de Sta. Rosa, antes de 1739

Inácio de $\mathrm{S}$. José, $\$ 1747$

João de Sta. Teresa de Jesus Marís, 1752

Manuel de Sta. Clara, 1755/6

Domingos da Solidade, 1756

Francisco de Sta. Eufrásia Landim, 1757/60 (43)

Benedito de S. José, 1760

José da Conceição, $\$ 1760$.

(41). - Documentos Históricos, vol. 40, p. 334.

(42) . - Jaboatão I, 2 p. 115.

(43). - Studart fl. $6 r$. 
20). - Una-PE. 1679-1742.

Luís da Anunciação, cerca de 1600 (44)

João da Assunção, cerca de 1600 (45)

Manuel das Chagas I, (M) 1681

Lourenço de Jesus Maria, (C) 1681

Manuel dos Santos, (M) 1697

José da Vịsitação, (C) 1697

Plácido da Purificação, $† 1727$

José do Desterro, 1728

Lino da Cruz, 1735, 1738.

21). - Unhunhum-PE. 1705-1761.

Pantaleão da Porciúncula, 1715

Manuel de Sta. Joana, sem data

Alberto da Conceição, 1735

Antônio da Porciúncula I, 1738

Salvador de Sta. Maria, antes de 1739

José de Sta. Cipriana, $\nmid 1750$

Maurício de S. Francisco Macedo, 1758/60 (46).

$N B$ : Dos seguintes missionários não constam dados exatos: Fr. Felipe Benício passou três anos em Saí, tendo falecido no convento de Cairu-BA, em 1781; Frei Cosme de São José foi missionário de Coripós, Juazeiro e Pontal, durante $71 / 2$ anos. Faleceram em missões, sem constar onde, Fr. Antônio da Madre de Deus Caminha, em 1732, Fr. Francisco do Sepulcro, em 1737, e Fr. Antônio de Jesus Maria Couto, em 1751. Fr. João de Sta. Teresa passou três anos em Juazeiro e Pajeu. Faltam quaisquer indicações de missionários das aldeias de Piagui-BA, Cariris-PB e Camamu-BA. Não constam no arquivo franciscano as aldeias de Catu-BA e Moibis, com o missionário desta, Frei Miguel da Conceição, em 1688. As missões de Arachá e Cararu tiveram administração franciscana interina, por volta de 1700 .

\section{Conclusão.}

As missões entre os índios ocupam as páginas mais brilhantes da história franciscana. Pois, a introdução dos nossos silvícolas na re-

(44) . - Jaboatão I, 2 p. 323s. Una foi a única missão curada pelos franciscanos, durante o tempo da custódia e da província, razão por que a incluimos aqui.

(45). - Ibidem, p. 323s.

(46). - Studart fl. 24r. 
ligião católica e na civilização constituiu sempre a primeira preocupação dos filhos de São Francisco, desde o descobrimento do Brasil.

O elenco dos missionários deixa entrever o espírito elevado dos apóstolos da selva que trocaram o relativo, conforto claustral com os labores e sacrifícios dos evangelizadores, embrenhando-se nos adustos sertões. Eis os religiosos que se destacaram pelo apostolado prolongado: Frei José da Encarnação Silvestre passou 49 anos entre os seus aldeados; Frei Antônio do Desterro Padilha, 38 anos; Frei Antônio de Santa Clara, 37 anos; Frei Joaquim de São José e Santana, 27 anos; Frei Sebastião de Santo Antônio Marques e Frei Francisco de São Sebastiẫo, cada um 24 anos; Frei Florentino da Sagrada Família, 21 anos. Tais fatos dispensam comentários.

Para termos uma idéia das penosas viagens missionárias, basta lembrar que o percurso de Salvador até Juazeiro pedia um mês, visto que os franciscanos pela regra seráfica, eram proibidos de montar a cavalo. Frei João de Santana, que em 1800 se submeteu à mesma jornada, faleceu logo que chegou a Juazeiro.

A Província de Santo Antônio incumbiu-se das missões durante dois séculos, cedendo de bom grado os melhores frades e enfrentando mil dificuldades, na defesa dos índios confiados à sua tutela .

Infelizmente a história missionária do Brasil que vem a ser a mais importante não encontrou ainda o seu autor. Enquanto ela nos faltar ficará incompleta a verdadeira história do País. 